\title{
Chemical and Microbiological Comparisons of Inhibitors Derived Thermally from Nitrite with an Iron Thionitrosyl (Roussin Black Salt)
}

\author{
By J. ASHWORTH, A. DIDCOCK, LINDA L. HARGREAVES, \\ B. JARVIS AND C. L. WALTERS \\ British Food Manufacturing Industries Research Association, \\ Leatherhead, Surrey, $K_{22}{ }_{7} R Y$ \\ AND L. F. LARKWORTHY \\ Department of Chemistry, University of Surrey, Guildford $\mathrm{GU}_{2}{ }_{5} \mathrm{XH}$
}

(Received 20 May 1974)

\section{INTRODUCTION}

In 1967 , Perigo, Whiting \& Bashford reported that nitrite heated in a complex laboratory medium was more inhibitory to Clostridium sporogenes than was unheated nitrite, and that the inhibitory activity of this heated nitrite system differed in several respects from a system containing unheated nitrite. These observations have been subsequently confirmed by Perigo \& Roberts (1968), and by Johnston, Pivnick \& Samson (1969).

Perigo \& Roberts (I968) examined the inhibitory activity of heated systems of media and nitrite against a range of clostridia, and showed that the thermally induced inhibitor (Perigo type factor; PTF) was effective against a number of strains of Clostridium botulinum (types A, B, E and F), Cl. welchii, Cl. tetani, Cl. bifermentans, Cl. septicum and $\mathrm{Cl}$. histolyticum. In addition Roberts \& Garcia (1973) have shown that whilst the PTF is effective against some Bacillus species, other strains of Bacillus and several strains of Salmonella were resistant to the inhibitor.

Investigations have been made into the role of PTFs in both meat and culture-medium systems which have been given either sterilization or pasteurization heat treatments (Ashworth \& Spencer, 1972; Ashworth, Hargreaves \& Jarvis, 1973). Preliminary chemical characterization of the PTF from various culture medium systems indicated some similarity with a range of iron nitrosyl co-ordination complexes described originally by Roussin ( $\left.185^{8}\right)$. The antimicrobial activity of tri- $\mu_{3}$-thio-mononitrosyl-tris(dinitrosyl)tetraferrate (Roussin black salt) has been studied previously against a range of micro-organisms (Dobry \& Boyer, I944; Candeli \& Mancini, 1948; Candeli, 1949). During the course of our own work it was necessary to develop a rapid and sensitive microbiological assay for the PTF (Ashworth, Hargreaves, Rosser \& Jarvis, 1974). This paper reports studies of the chemical properties and the antimicrobial spectrum of Roussin black salt and of the PTFs from various culturemedia systems.

\section{METHODS}

Organisms. The organisms tested are listed in Table I together with details of the temperature of incubation. Cultures were maintained on slopes of nutrient agar, except for $\mathrm{Cl}$. sporogenes which was maintained in cooked meat broth (Southern Group Laboratories, 
Table I. Antimicrobial spectrum of the inhibitory systems and Roussin black salt

\begin{tabular}{|c|c|c|c|c|c|c|c|c|c|c|c|c|c|c|}
\hline & & \multicolumn{13}{|c|}{ Inhibition* caused by } \\
\hline Organism & $\begin{array}{c}\text { Temperature } \\
\text { of } \\
\text { incubation } \\
\left({ }^{\circ} \mathrm{C}\right)\end{array}$ & 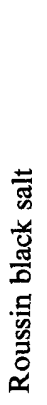 & 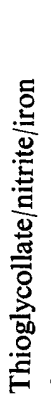 & 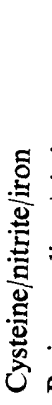 & 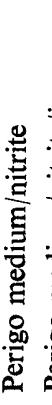 & 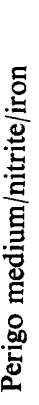 & 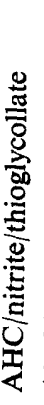 & 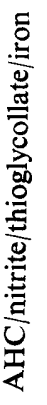 & 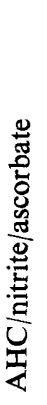 & 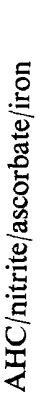 & 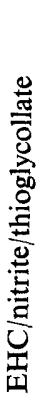 & 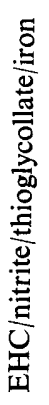 & 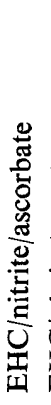 & 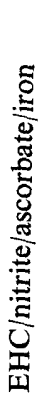 \\
\hline ilis ATcc6633 & 30 & 3 & 3 & 3 & I & 3 & 2 & 2 & $\mathbf{I}$ & 2 & 2 & 2 & 2 & 3 \\
\hline rC2599 & 37 & 2 & 2 & 3 & I & 1 & $\mathbf{I}$ & 2 & I & 2 & 2 & $\mathbf{I}$ & 2 & 2 \\
\hline porogenes NCTC8594 & 37 & 4 & 4 & 3 & 0 & I & 1 & 2 & I & 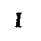 & 1 & 3 & I & 3 \\
\hline ATCC934I & 30 & 3 & 3 & 3 & $\mathbf{I}$ & 2 & 2 & 3 & $\mathbf{I}$ & 2 & 2 & 2 & 2 & 3 \\
\hline cus saprophyticus NCTC729I & 37 & 3 & 3 & 3 & I & 2 & I & 3 & $\mathbf{I}$ & 2 & 2 & $\mathbf{I}$ & 2 & 2 \\
\hline $\mathrm{TC} 8532$ & 37 & 3 & 2 & 2 & 1 & 2 & 2 & 2 & $\mathbf{I}$ & 2 & I & $\mathbf{I}$ & 2 & 2 \\
\hline$s$ brevis FRAI076† & 30 & I & 1 & I & I & I & I & I & I & I & $\mathbf{I}$ & I & I & $\mathbf{I}$ \\
\hline FRAI077 $\dagger$ & 30 & $\mathbf{I}$ & $\mathbf{I}$ & 0 & 0 & I & I & I & I & I & 0 & o & o & 0 \\
\hline is durans $\mathrm{c} 3 \ddagger$ & 37 & 2 & 2 & 2 & 0 & I & I & 2 & o & 2 & I & 2 & I & I \\
\hline s oleovorans NCTC 6576 & 37 & 2 & 2 & 2 & 0 & I & I & 2 & 0 & I & 0 & 2 & 0 & I \\
\hline sp. FRAIO78 $\uparrow$ & 37 & 2 & 2 & I & 0 & I & 0 & I & 0 & I & I & I & I & I \\
\hline FRAIO79 & 37 & 2 & 2 & 2 & 0 & 0 & 0 & I & 0 & I & I & I & I & $\mathbf{I}$ \\
\hline rogenes NCTC8I 72 & 37 & 2 & 1 & 2 & 0 & I & 0 & 1 & 0 & $\mathbf{I}$ & 0 & I & I & I \\
\hline cescens NCTCI 377 & 30 & 2 & 2 & $\mathbf{I}$ & 0 & o & 0 & I & 0 & 0 & 0 & 0 & 0 & 0 \\
\hline coli NCTC8I96 & 37 & 2 & I & 1 & 0 & 0 & o & I & 0 & $\mathbf{I}$ & 0 & $\mathbf{I}$ & 0 & 0 \\
\hline yphimurium 7M4987§ & 37 & I & I & $\mathbf{I}$ & 0 & ० & 0 & $\mathbf{I}$ & 0 & o & o & $\mathbf{I}$ & 0 & I \\
\hline NCTC9959 & 37 & $\mathbf{I}$ & $\mathbf{I}$ & I & o & 0 & 0 & $\mathbf{I}$ & o & $\mathbf{I}$ & o & $\mathbf{I}$ & 0 & $\mathbf{I}$ \\
\hline freundii NCTC81 65 & 37 & I & $\mathbf{I}$ & 0 & 0 & 0 & 0 & I & o & o & 0 & 0 & 0 & 0 \\
\hline is NCYC359 & 30 & 2 & 3 & 2 & 0 & I & $\mathbf{I}$ & 2 & 0 & I & o & $\mathbf{I}$ & $\mathbf{I}$ & 2 \\
\hline
\end{tabular}

Bacillus subtilis ATcc6633

B. cereus NCTC2599

Clostridium sporogenes NCTC8594

Sarcinia lutea ATCC9341

Staphylococcus saprophyticus NCTC729I

$S$. aureus NCTC8532

Lactobacillus brevis FRA1076

L. buchneri FRAI077†

Streptococcus durans $\mathrm{C} 3 \ddagger$

Pseudomonas oleovorans NCTC6576

Providencia sp. FRA1078

Proteus sp. FRAI079†

Klebsiella aerogenes NCTC8I72

Serratia marcescens NCTCI 377

Escherichia coli NCTC8196

Salmonella typhimurium 7M49878

S. senftenberg NCTC9959

Citrobacter freundii NCTC81 65

Candida utilis NCYC359

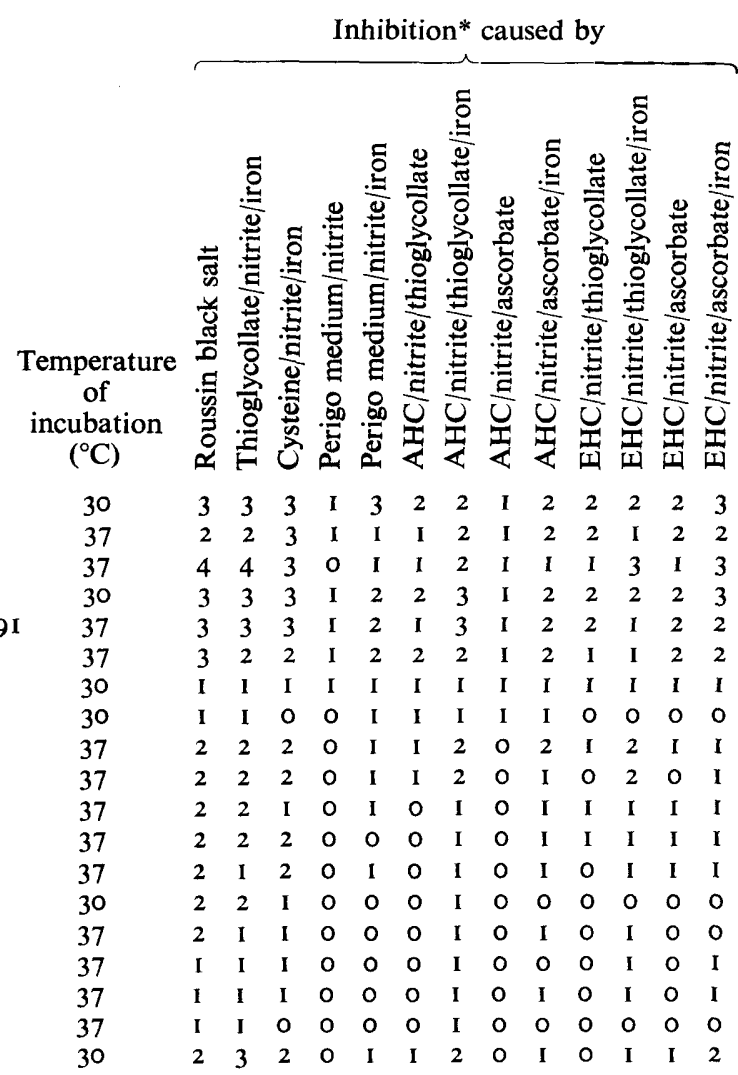

* Symbols for linear inhibition (mm): (o), none; (1), o to $2 \cdot 9 ;(2), 3$ to $5 \cdot 9 ;(3), 6$ to $8 \cdot 9 ;$ (4), 9 to I2.

$\dagger$ Food Research Association culture collection.

$\ddagger$ Kindly supplied by Dr E. M. Barnes, Food Research Institute, Norwich.

$\S$ Kindly supplied by Dr E. S. Anderson, Enteric Reference Laboratory, Central Public Health Laboratory, Colindale, London.

London), lactobacilli in Lactobacillus MRS broth (Difco) and Candida utilis on malt extract agar (Oxoid). Test plates were inoculated with $18 \mathrm{~h}$ broth cultures of the organisms.

Preparation of inhibitors. (i) Potassium and ammonium Roussin black salts were prepared by the method of Seel (1965). The product was recrystallized from hot $\left(70^{\circ} \mathrm{C}\right)$ distilled water containing a few drops of alkali and stored in vacuo at $-35{ }^{\circ} \mathrm{C}$ in the dark.

(ii) Inhibitors from thioglycollate or cysteine $(0.2 \%, \mathrm{w} / \mathrm{v})$ were prepared by heating with 500 p.p.m. ferrous sulphate and 100 p.p.m. sodium nitrite (final concentrations) at $115{ }^{\circ} \mathrm{C}$ for $20 \mathrm{~min}$.

(iii) Inhibitors from 'Perigo' medium. This medium, prepared as described by Perigo et al. (1967), was heated at I $1{ }^{\circ} \mathrm{C}$ for 20 min with 100 p.p.m. sodium nitrite, with or without 500 p.p.m. ferrous sulphate.

(iv) Inhibitors from casein hydrolysates. A solution of $15 \%(\mathrm{w} / \mathrm{v})$ acid hydrolysed casein (AHC; Sigma) was heated ( $115{ }^{\circ} \mathrm{C}$ for $20 \mathrm{~min}$ ) with 100 p.p.m. sodium nitrite and $0.2 \%$ $(w / v)$ of either sodium thioglycollate or sodium ascorbate, with and without the addition 
of 500 p.p.m. ferrous sulphate. A $10 \%(w / v)$ solution of enzymic hydrolysed casein (EHC; Sigma) was given a similar treatment.

Extraction of inhibitors. After the heat treatment with nitrite the systems examined were cooled, and were then extracted three times by shaking with $0 \cdot$ I vol. of diethyl ether (Fisons Chemicals, analytical grade). The other extracts were combined, washed with distilled water, and evaporated to dryness in vacuo in a rotary evaporator. Samples were purified by thin-layer chromatography in $250 \mu \mathrm{m}$ thick silica gel ' $\mathrm{GF}$ ' plates (Analtech Inc.), using an acetone - petroleum ether $(3: 2)$ mixture.

Nitrosamines. $N$-nitrosoproline and $N$-nitrososarcosine were prepared by the method of Stewart (1969). $N$-nitrosodimethylamine, $N$-nitrosodiethylamine and $N$-nitrosopiperidine were obtained from Eastman Kodak Inc.

Chemical analysis. Infrared spectra were obtained by using either a Unicam SP200 or a Perkin Elmer 457 recording spectrometer. Samples were examined either in chloroform solution in cells of I mm path length, or as nujol mulls. Ultraviolet and visible spectra of aqueous solutions of the samples, in $10 \mathrm{~mm}$ silica cells, were determined with a Unicam SP800 spectrophotometer. ${ }^{57} \mathrm{Fe}$ Mössbauer data (Herber, 197I) were obtained at room temperature using ${ }^{57} \mathrm{Co}$ in a matrix of palladium as the gamma radiation source.

General procedure for the determination of antimicrobial spectra of the inhibitors. One well of $16 \mathrm{~mm}$ diam was punched in the centre of each of a series of $8.5 \mathrm{~cm}$ diam plates containing $23 \mathrm{ml}$ nutrient agar (Oxoid), previously air dried at $37^{\circ} \mathrm{C}$ for $3 \mathrm{~h}$. Broth cultures $(\mathrm{I} 8 \mathrm{~h})$ of the various organisms to be examined (generally in nutrient broth) were streaked radially on to the plates from the periphery of the plate to the edge of the well. Normally six organisms were streaked on a plate, but the Proteus species was cultivated individually.

Clostridium sporogenes was grown in cooked meat broth and streaked on plates of reinforced clostridial agar (Oxoid); the lactobacilli were streaked on Lactobacillus MRS broth solidified with $\mathrm{I} \cdot 5 \%(\mathrm{w} / \mathrm{v})$ agar.

Aqueous solutions (100 p.p.m.) of the inhibitors were introduced into the well $(0.4 \mathrm{ml})$ in each plate, and the plates were then incubated overnight at the appropriate temperature. The lengths of the zones of inhibition were measured for each organism tested.

\section{RESULTS AND DISCUSSION}

Examination of the physico-chemical properties of the Roussin black salt, and the various inhibitors extracted from systems of media heated with nitrite, reveal many similarities. The infrared spectrum of ammonium Roussin black salt showed a medium-strength absorption peak at $1604 \mathrm{~cm}^{-1}$, a strong broad peak at $1752 \mathrm{~cm}^{-1}$ with a shoulder at $1716 \mathrm{~cm}^{-1}$, and a medium-strength peak at $1802 \mathrm{~cm}^{-1}$; the absorption spectrum of the potassium salt differed only in that a shoulder was seen at $1749 \mathrm{~cm}^{-1}$, and none at $1716 \mathrm{~cm}^{-1}$. All extracts of the various inhibitory systems examined characteristically showed peaks in the regions I603 to I604, I752 to I 753 , and 1802 to $1803 \mathrm{~cm}^{-1}$. Except for the AHC/ascorbate/nitrite/ iron system, a shoulder was observed at 1712 to $I 7 I 4 \mathrm{~cm}^{-1}$. An extract of a non-inhibitory system of AHC and ascorbate had weak absorption peaks at 1602 and $1680 \mathrm{~cm}^{-1}$ only. Thus there was a considerable similarity between the i.r. absorption spectra of inhibitory EHC and AHC extracts and those of the potassium and ammonium Roussin black salts. The absorption peak at $1750 \mathrm{~cm}^{-1}$ is typical of the $\mathrm{N}-\mathrm{O}$ stretch. The u.v. absorption spectra of freshly prepared inhibitors from AHC and EHC systems heated with nitrite were similar to those obtained for Roussin black salts, and for inhibitors obtained from cysteine or 
thioglycollate heated with ascorbate and nitrite. Absorption with broad maxima of low intensity occurred in the regions 260 to $270 \mathrm{~nm}$ and 420 to $430 \mathrm{~nm}$. On storage in the light at room temperature similar changes in u.v. and i.r. spectra were observed for Roussin black salt and for the inhibitory extracts.

The Mössbauer spectra of potassium and ammonium Roussin black salts, and for the inhibitor extracted from a heated AHC/ascorbate/nitrite system, revealed isomer shift $(\delta)$ values of $0.04,0.04$ and $0.1 \mathrm{~mm} / \mathrm{s}$ respectively. These values are of a similar order to the value of $0.07 \mathrm{~mm} / \mathrm{s}$ reported for sodium Roussin black salt (Kerler et al. I963). Quadrupole split $\left(\Delta E_{Q}\right)$ for the potassium and ammonium Roussin black salts and the AHC inhibitor were found to be $0.90,0.80$ and $0.60 \mathrm{~mm} / \mathrm{s}$ respectively; the published value for sodium Roussin black salt is $0.87 \mathrm{~mm} / \mathrm{s}$ (Kerler et al. 1963). The Mössbauer spectrum of the inhibitor obtained from the AHC system clearly shows that this compound is not identical with the potassium, sodium, or ammonium Roussin black salts. However, since the $\Delta E_{Q}$ (quadrupole splitting) measurements of the potassium and ammonium salts are 0.9 and $0.8 \mathrm{~mm} / \mathrm{s}$ respectively, it is obvious that the cation has an influence upon the $\Delta E_{Q}$ values.

Elemental analysis of a diethyl ether extract from the AHC/ascorbate/nitrite system gave the values: $\mathrm{Fe}, 42 \cdot \mathrm{I} ; \mathrm{S}, \mathrm{I} 8 \cdot \mathrm{I} ; \mathrm{N}, \mathrm{I} 6 \cdot \mathrm{I} ; \mathrm{O}, 23.8 ; \mathrm{H},<\mathrm{I} \%$. Calculated values for ammonium Roussin black salts are: $\mathrm{Fe}, 40 \cdot 8 ; \mathrm{S}, \mathrm{I} 7 \cdot 6 ; \mathrm{N}, 20.5 ; \mathrm{O}, 20 \cdot 5 ; \mathrm{H}, 0.7 \%$, with similar values being obtained by calculation for sodium and potassium Roussin black salts. The elementary analysis of the AHC extract is therefore very similar to the theoretical elemental composition of Roussin black salts. The $R_{F}$ values of the inhibitory fraction from AHC systems and of potassium Roussin black salt separated on silica gel GF by means of acetone/petroleum ether were similar. The evidence strongly suggests a degree of chemical similarity between the inhibitor extracted from a system of AHC heated with nitrite and Roussin black salts, although the Mössbauer spectra suggest differences, possibly in the cation moiety.

\section{Antimicrobial spectrum}

The antimicrobial spectra of ammonium Roussin black salt and of the thermally-induced inhibitors derived from nitrite, against a range of micro-organisms, are shown in Table I. The five $N$-nitrosamines tested had no inhibitory effect against any of the test cultures at I000 p.p.m. When a system of medium and nitrite was heated with ionic iron, there was always a statistically significant increase in the inhibitory activity of the system, without necessarily a change in the antimicrobial spectrum $(P<0.005$ with 8 degrees of freedom). There was, however, no statistically significant difference between the activity and spectra of inhibitory systems containing thioglycollate and those containing ascorbate as reducing agents. The antimicrobial spectra of the various systems showed that the inhibitors were more active against the Gram-positive than the Gram-negative bacteria tested $(P<0.00 I$ with 16 degrees of freedom).

The production of the PTF has also been demonstrated in systems of whole pork and nitrite, given either a sterilization heat treatment (Ashworth \& Spencer, 1972) or a pasteurization heat treatment (Ashworth et al. 1973). Attempts to examine the antimicrobial spectrum and chemical characteristics of PTFs from meat systems have been unsuccessful, mainly because of the inability to extract these inhibitors from the meat systems.

The antimicrobial spectrum of ammonium Roussin black salt reported here, is basically similar to the results obtained by Candeli \& Mancini (1948) except for Serratia marcescens, for which the differing response might be explained by interstrain variation. The antimicrobial spectrum of the cysteine/nitrite/iron system against Clostridium sporogenes, 
Salmonella spp., and Streptococcus-sp. is not dissimilar to the antimicrobial activity of cysteine nitrosothiol reported by Incke, Farkas, Mihályi \& Zukal (1974). Roberts \& Garcia (1973) examined the inhibitory activity of Perigo medium (Perigo et al. 1967) heated with nitrite, and found that in addition to a range of clostridia, the system was active against many Bacillus spp. tested, including B. subtilis, and against Streptococcus faecium and Streptococcus faecalis, but inactive against strains of Salmonella typhimurium. The findings reported here for the same medium are basically in accord with these authors' results.

The potency of the inhibitors derived from systems of the original medium of Perigo et al. (1967) and all the other systems tested, with the exception of AHC heated with nitrite and ascorbate, is considerably less than that of ammonium Roussin black salt. In many of the systems tested it is possible that the extracts were impure, and that this could lead to an apparent diminution in the activity of the inhibitory extract. This, together with the observed reduced stability of the extracted inhibitors compared to Roussin black salt, may partially explain the difference found between the systems tested. The results show that small quantities of inhibitor are produced from the Perigo medium, AHC and EHC, without the addition of iron salts, but the addition of iron salts increases the quantity of inhibitor produced in the various systems. This is in agreement with the results of elemental analysis, i.e. the inhibitors are iron-containing substances. There is, however, sufficient iron in the Perigo medium, AHC, or EHC to permit the formation of small amounts of inhibitor even when iron salts are not added.

Wasserman \& Huhtanen (1972) showed that the Perigo type inhibitor (PTF) did not appear to be a nitrosamine, and they failed to demonstrate any inhibitory effect against Clostridium botulinum by a range of $N$-nitrosamines. This result is confirmed in the present work where none of the $N$-nitrosamines tested showed any inhibitor activity.

The work reported here shows that a potent antimicrobial substance with a broad spectrum is formed when nitrite is heated in certain systems, and that this inhibitory substance is similar in many respects to the iron nitrosyl co-ordination complexes, termed Roussin black salts.

We thank Miss Anthea Rosser and Miss Amanda Walker for excellent assistance during part of the experimental work, and Dr B. Fitzsimmons, Birkbeck College, University of London, for determination of Mössbauer spectra.

\section{REFERENCES}

Ashworth, J., Hargreaves, L. L. \& Jarvis, B. (1973). The production of an antimicrobial effect in pork heated with sodium nitrite under simulated commercial pasteurization conditions. Journal of Food Technology 8, 477-484.

Ashworth, J., HARgreaves, L. L., Rosser, A. \& JARVis, B. (1974). The assay of thermally induced inhibitors derived from nitrite. Society for Applied Bacteriology Technical Series 8, 75-90.

Ashworth, J. \& SPENCER, R. (1972). The Perigo effect in pork. Journal of Food Technology 7, I I I-I 24.

CANDEli, A. (1949). Meccanismo dell'azione antibatterica del ferro-eptanitroso-trisolfuro di potassio. Bollettino della Società italiana di biologia sperimentale 25, 495-497.

Candeli, A. \& Mancini, M. (1948). Azione batteriostatica del ferro-eptanitroso-trisolfuro di potassio. Bollettino della Società medico-chirurgica di Modena 48, 16I-165.

Dobry, A. \& Boyer, F. (I944). Sur le nitrososulfure de fer ou sel de Roussin action antiseptique. Annales de l'Institut Pasteur 71, 455-462.

Herber, R. H. (I97I). Mössbauer spectroscopy. Scientific American 225 (4), 86-95.

INCKe, K., Farkas, J., Mihályi, V. \& Zukal, E. (1974). Antibacterial effect of cysteine-nitrosothiol and possible percursors thereof. Applied Microbiology 27, 202-205. 
Johnston, M. A., Pivnick, H. \& Samson, J. M. (1969). Inhibition of Clostridium botulinum by sodium nitrite in a bacteriological medium and in meat. Canadian Institute of Food Science and Technology Journal 2, 52-55.

Kerler, W., Neuwirth, W., Fluck, E., Kuhn, P. \& ZimmermanN, B. (1963). Untersuchung komplexer und kovalenter Eisenverbindungen mit Hilfe des Mössbauer - Effects von Fe ${ }^{57}$. Zeitschrift für Physik I73, $32 \mathrm{I}-346$.

Perigo, J. A. \& Roberts, T. A. (1968). Inhibition of clostridia by nitrite. Journal of Food Technology 3 , 9I-94.

Perigo, J. A., Whiting, E. \& Bashford, T. E. (1967). Observations on the inhibition of vegetative cells of Clostridium sporogenes by nitrite, which has been autoclaved in a laboratory medium, discussed in the context of sublethally processed cured meats. Journal of Food Technology 2, 377-397.

RoberTs, T. A. \& GARCIA, C. E. (1973). A note on the resistance of Bacillus spp., faecal streptococci, and Salmonella typhimurium to an inhibitor of Clostridium spp. formed by heating sodium nitrite. Journal of Food Technology 8, 463-466.

Roussin, M. L. (1858). Recherches sur les nitrosulfures double de fer. Annales de chimie et de physique $\mathbf{5 2}^{2}$, 285-303.

SeEL, F. (1965). Carbonyl and nitrosyl compounds. In Handbook of Preparative Inorganic Chemistry, 2nd edn, p. I764. Edited by G. Brauer. London and New York: Academic Press.

Stewart, F. H. C. (I969). Peptide synthesis with the $N$-nitroso derivatives of sarcosine and proline. Australian Journal of Chemistry 22, 245I-246I.

Wasserman, A. E. \& Huhtanen, C. N. (1972). Nitrosamines and the inhibition of Clostridia in medium heated with sodium nitrite. Journal of Food Science 37, 785-786. 